\title{
Optimizing the magnetic dimensions for maximum thrust in high density transverse flux linear motor
}

\begin{abstract}
This paper describes the optimization of the magnetic dimensions for maximum thrust for a High Thrust Density Transverse Flux Linear Motor (HDTFLM). HDTFLM is a novel structure with combination of a typical High Thrust Density Linear Motor (HDLM) and Transverse Flux Linear Motor (TFLM) structure. The magnetic circuit of HDTFLM is different compared to conventional three phase linear motor because it has independences magnetic circuit on each phase. The optimization of maximum thrust is achieved by varying the thickness and height of the permanent magnet. This paper reports the optimized dimensional values for the HDTFLM structure for maximum thrust development.
\end{abstract}

Keyword: High thrust density; Linear motor; Permanent magnet; Transverse flux 